\title{
THE BNL RARE KAON DECAY PROGRAM*
}

\author{
Laurence Littenberg \\ Physics 510 A \\ Brookhaven National Laboratory \\ Upton, NY 11973-5000
}

\begin{abstract}
The rare kaon decay program at Brookhaven National Laboratory is reviewed. Results from the last round of experiments are briefly discussed. The three experiments currently collecting data are described. Prospects for future experiments are discussed.
\end{abstract}
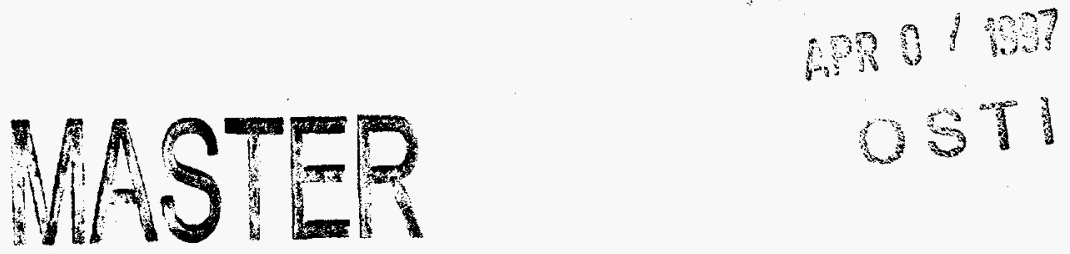

* To be published in the Proceedings of the XXIV SLAC Summer Institute on Particle Physics 


\section{DISCLAIMER}

This report was prepared as an account of work sponsored by an agency of the United States Government. Neither the United States Government nor any agency thereof, nor any of their employees, make any warranty, express or implied, or assumes any legal liability or responsibility for the accuracy, completeness, or usefulness of any information, apparatus, product, or process disclosed, or represents that its use would not infringe privately owned rights. Reference herein to any specific commercial product, process, or service by trade name, trademark, manufacturer, or otherwise does not necessarily constitute or imply its endorsement, recommendation, or favoring by the United States Government or any agency thereof. The views and opinions of authors expressed herein do not necessarily state or reflect those of the United States Government or any agency thereof. 


\section{DISCLAMMER}

Portions of this document may be illegible in electronic image products. Images are produced from the best available original document. 


\section{Introduction}

After more than a decade of "benign neglect", in which there were no rare $K$ decay experiments at Brookhaven, a new round of experiments was launched in the early 1980's. The motivation for this program was mainly the search for physics beyond the Standard Model (SM). ${ }^{1}$ It had been realized in the late 1970's that "lepton flavor violating" (LFV) processes such as $\mu \rightarrow e \gamma$ and $K_{L} \rightarrow \mu e$ arise naturally in theoretical attempts to go beyond the Standard Model ${ }^{2}$ (BSM). Moreover, it turned out that kaon branching ratios accessible at the AGS correspond to extremely high energy scales for possible new interactions. The classic example is the latter process, $K_{L} \rightarrow \mu e$, which in Fig. 1 is shown mediated by a hypothetical horizontal gauge boson, and compared to the kinematically very similar $K^{+} \rightarrow$ $\mu^{+} \nu$ decay.
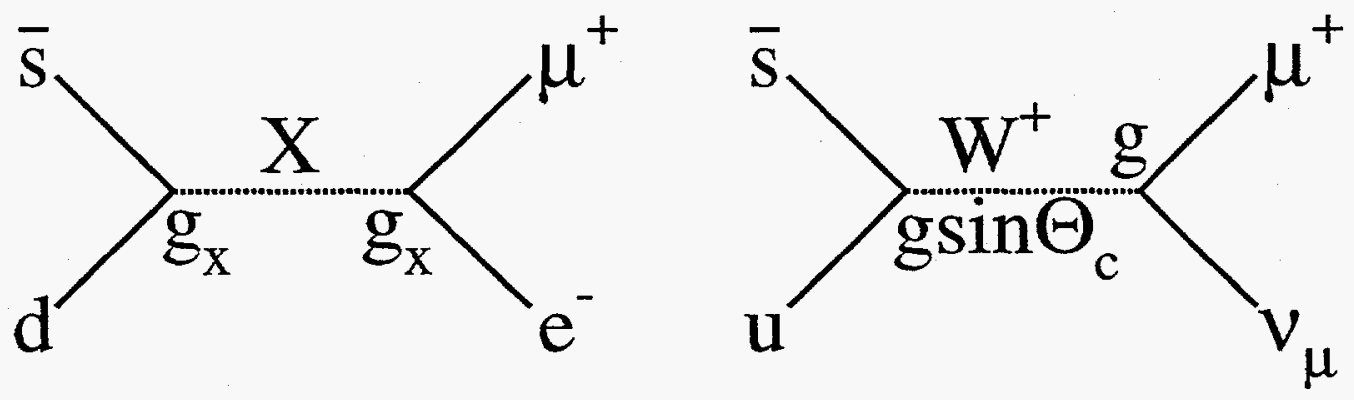

Figure 1: Horizontal gauge boson mediating $K_{L} \rightarrow \mu e$, compared with $W$ mediating $K^{+} \rightarrow \mu^{+} \nu$.

Using measured values for $M_{W}$, the $K_{L}$ and $K^{+}$decay rates and $B\left(K^{+} \rightarrow \mu^{+} \nu\right)$, and assuming a $V-A$ form for the new interaction, one can show

$$
M_{X} \approx 220 \mathrm{TeV} / c^{2}\left[\frac{g_{X}}{g}\right]^{1 / 4}\left[\frac{10^{-12}}{B\left(K_{L} \rightarrow \mu e\right)}\right]^{1 / 4}
$$

so that truly formidable scales can be probed if $g_{X} \sim g$. For a $V-A$ interaction, $K_{L} \rightarrow \mu e$ is the most sensitive of kaon decays. If, however, the LFV interaction were pure vector instead, the two-body reaction would be suppressed, and one would be better off searching for $K^{+} \rightarrow \pi^{+} \mu^{+} e^{-}$, a reaction that also has some advantages in signature with respect to $K_{L} \rightarrow \mu e$. One could also substitute neutral for charged leptons in this last reaction and search for $K^{+} \rightarrow \pi^{+} \nu_{e} \bar{\nu}_{\mu}$. Although it has a much worse signature than the decays into charged leptons, a search for this process is also sensitive to the considerable number of proposed 
BSM processes whose topology is $K^{+} \rightarrow \pi^{+}+$nothing (where nothing denotes an undetectable light particle or system of particles ${ }^{3,4}$ ). Of course one member of this family, $K^{+} \rightarrow \pi^{+} \nu_{\ell} \bar{\nu}_{\ell}$, is allowed in the SM, but it is suppressed far beyond the experimental limits current in the early 1980 's. ${ }^{5}$

Other experiments in this program probed non-SM CP-violating reactions, or searched for a light Higgs or for unexpected enhancements in SM-suppressed decays such as $K_{L} \rightarrow e^{+} e^{-}$. Most of the experiments were also capable of studying somewhat more common $K$ decays, and so were able to contribute to the development of new theoretical approaches to long distance physics, most notably Chiral Perturbation Theory ${ }^{6}(\chi P T)$.

Table 1 lists the first round experiments. These completed data-taking by 1991.

Table 1: First round experiments of the BNL Rare $K$ Decay Program. Those denoted by a star were 'descendents' of the experiment directly above them in the Table.

\begin{tabular}{|c|c|c|}
\hline EXPERIMENT & MODES & INSTITUTIONS \\
\hline AGS-780 & $K_{L} \rightarrow \mu e, e e, \mu \mu, \pi^{0} e^{+} e^{-}$ & $\overline{B N L}$, Yale \\
\hline AGS-845* & $K_{L} \rightarrow \pi^{0} e^{+} e^{-}, \gamma e^{+} e^{-}, e^{+} e^{-} e^{+} e^{-}$ & BNL, Yale \\
\hline AGS-777 & $K^{+} \rightarrow \pi^{+} e^{-} \mu^{+}, \pi^{+} e^{+} e^{-}, \ldots$ & $\begin{array}{c}\text { BNL, SIN, } \\
\text { Washington, Yale }\end{array}$ \\
\hline AGS-851* & $K^{+} \rightarrow \pi^{+} e^{+} e^{-}, \pi^{0} \rightarrow e^{+} e^{-}$ & $\begin{array}{c}\text { BNL, SIN, } \\
\text { Washington, Yale }\end{array}$ \\
\hline AGS-791 & $K_{L} \rightarrow \mu e, e e, \mu \mu, \ldots$ & $\begin{array}{l}\text { UCI, UCLA, LANL } \\
\text { Stanford, Temple } \\
\text { U. of Texas Austin, } \\
\text { William \& Mary }\end{array}$ \\
\hline AGS-787 & $K^{+} \rightarrow \pi^{+} \nu \bar{\nu}, \pi^{+} \mu^{+} \mu^{-}, \ldots$ & $\begin{array}{l}\text { BNL, Princeton } \\
\text { TRIUMF }\end{array}$ \\
\hline
\end{tabular}

Table 2 summarizes the achievements of the first round of experiments. Three very interesting decay modes were seen for the first time: $K_{L} \rightarrow \gamma \gamma e^{+} e^{-}, K^{+} \rightarrow$ $\pi^{+} \mu^{+} \mu^{-}$, and $K^{+} \rightarrow \pi^{+} \gamma \gamma$. The available data sets of other interesting processes $\left(K^{+} \rightarrow \pi^{+} e^{+} e^{-}, 9 K_{L} \rightarrow \mu^{+} \mu^{-}, K_{L} \rightarrow \gamma e^{+} e^{-}\right.$, etc.) were very significantly increased, and new structure observed in the decay distributions of some of these. Finally, the limits on forbidden and suppressed processes were greatly improved, in a couple of cases by many orders of magnitude. 
Table 2: Results of the first round of the BNL Rare $K$ Decay Program.

\begin{tabular}{|l|c|c|}
\hline Mode & Result & Comment \\
\hline$K_{L} \rightarrow \mu e$ & BR $<3.3 \times 10^{-11} /$ evt & $\begin{array}{c}\text { BR limit improved by } 2 \times 10^{5} \\
M_{A}>94 \mathrm{TeV}\end{array}$ \\
\hline$K^{+} \rightarrow \pi \mu e$ & BR $<2.1 \times 10^{-11} /$ evt & $\begin{array}{c}\text { BR limit improved by } 22 \\
M_{V}>39 \mathrm{TeV}\end{array}$ \\
\hline$K^{+} \rightarrow \pi^{+} X^{0}$ & $\mathrm{BR}<5.2 \times 10^{-10}$ & $\begin{array}{c}\text { BR limit improved by } 70 \\
F_{K \pi}^{\text {familon }}>2.2 \times 10^{8} \mathrm{TeV}\end{array}$ \\
\hline$K^{+} \rightarrow \pi^{+} \nu \bar{\nu}$ & $\mathrm{BR}<2.4 \times 10^{-9}$ & BR limit improved by 60 \\
\hline$K_{L} \rightarrow \mu^{+} \mu^{-}$ & $>700$ evts & previous world supply 27 evts \\
\hline$K_{L} \rightarrow \pi^{0} e^{+} e^{-}$ & $\mathrm{BR}<5.5 \times 10^{-9}$ & BR limit improved by 360 \\
\hline$K_{L} \rightarrow e^{+} e^{-} \gamma \gamma$ & $(6.6 \pm 3.2) \times 10^{-7}$ & $\begin{array}{c}\text { Discovery, changed the course } \\
\text { of the study of CP-violation }\end{array}$ \\
\hline$K^{+} \rightarrow \pi^{+} e^{+} e^{-}$ & 512 evts & $\begin{array}{c}10 \text {-fold improvement, } \\
\text { form factor observed, } \\
\chi \mathrm{PT} \text { ambiguity broken }\end{array}$ \\
\hline$K_{L} \rightarrow \gamma e^{+} e^{-}$ & $\left(9.1 \pm 0.4_{-.5}^{+.6}\right) \times 10^{-6}$ & $\begin{array}{c}250 \text {-fold improvement, } \\
\text { form factor deviation observed }\end{array}$ \\
\hline$K^{+} \rightarrow \pi^{+} \mu^{+} \mu^{-}$ & & discovered \\
\hline$K^{+} \rightarrow \pi^{+} \gamma \gamma$ & & discovered \\
\hline$\pi^{0} \rightarrow e^{+} e^{-}$ & & cleared up a long-standing discrepancy \\
\hline
\end{tabular}

\section{The current experiments}

The three experiments which constitute the second generation of the AGS Rare Kaon Decay Program are listed in Table 3. Each is the successor of a first round experiment; in one case not even the name has changed. The motivation for these experiments has evolved since the first round was launched. The emphasis is now a little more on SM-suppressed processes and a little less on totally forbidden ones. This is largely because the advances in sensitivity achieved in the first round of experiments have brought some very interesting examples of the former processes into range. It has also been realized that kaon decays can contribute greatly to determining the CKM unitarity triangle. This is illustrated by Fig. 2 . All three sides of the triangle can in principle be determined from rare $K$ decays. In Fig. 2, the "primary" reactions are those adjacent to the sides of the triangle. The outlying reactions provide supplementary information needed for interpreting 
Table 3: Current experiments of the BNL Rare $K$ Decay Program.

\begin{tabular}{|c|c|c|}
\hline EXPERIMENT & MODES & INSTITUTIONS \\
\hline AGS-787 & $K^{+} \rightarrow \pi^{+} \nu \bar{\nu}, \pi^{+} X^{0}, \ldots$ & $\begin{array}{c}\text { BNL, INS/Tokyo, KEK } \\
\text { Osaka, Princeton, TRIUMF }\end{array}$ \\
\hline AGS-865 & $K^{+} \rightarrow \pi^{+} e^{-} \mu^{+}, \pi^{+} e e, \ldots$ & $\begin{array}{c}\text { Basel, BNL, } \\
\text { INR-Moscow, JINR-Dubna, } \\
\text { New Mexico, Pittsburgh, } \\
\text { PSI, Tbilisi State, } \\
\text { Yale, Zurich }\end{array}$ \\
\hline AGS-871 & $K_{L} \rightarrow \mu e, e e, \mu \mu, \ldots$ & $\begin{array}{c}\text { UCI, Stanford } \\
\text { U. of Texas Austin, } \\
\text { William \& Mary }\end{array}$ \\
\hline
\end{tabular}

some of the primary reactions, and for evaluating certain possible backgrounds.

Over the past few years, with the addition of a quarter-size booster and a revamped RF-system, the intensity of the AGS has steadily increased to the point where $6 \times 10^{13}$ protons/spill can routinely be accelerated to $25.2 \mathrm{GeV} / \mathrm{c}$. The $K$ experiments each utilize $1-2 \times 10^{13}$ protons/spill, which makes possible unprecedented sensitivities, but which has made necessary radiation-hard beam elements and innovations in production targets, trigger electronics and high rate detector design.

\section{$2.1 \quad$ E871}

E871 is designed to detect di-lepton decays of the $K_{L}$ at the $10^{-12}$ level. This requires a very large flux of $K_{L}$ and a detector that can make redundant high resolution measurements. Good particle identification power is also needed. The worst source of background to $K_{L} \rightarrow \mu e$ is $K_{L} \rightarrow \pi e \nu(K e 3)$. If the daughter pion decays in flight or is misidentified and the neutrino is soft, one has a background that appears topologically identical to the signal, with kinematics that can closely approach those of the signal. Since a few percent of the pions from $K e 3$ decay in the detector, particle identification per se is of limited value in this case. What is essential is excellent kinematic resolution and the ability to recognize track kinks. However if the $\mathrm{Ke} 3$ pion is mistaken for an electron and the electron for a muon, the reconstructed two-body mass can exceed (or equal!) $M_{K}$. In this case good kinematic resolution is of limited value but particle identification power is crucial. 
Rare $K$ Decays \& the Unitary Triangle

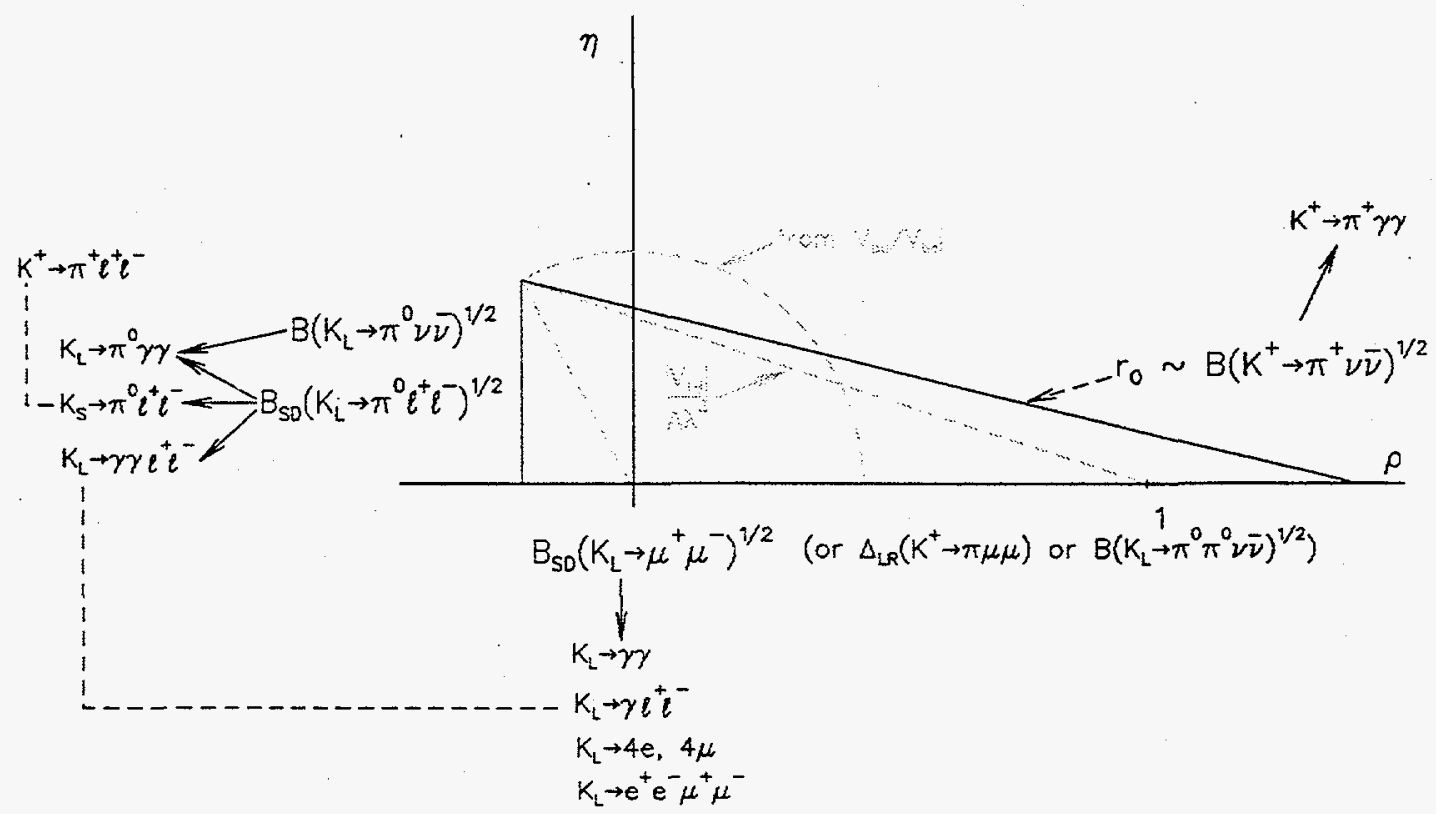

Figure 2: Rare $K$ decays and the unitarity triangle

The detector is shown in Fig. 3. An intense neutral beam containing $\sim 2 \times$ $10^{8} K_{L} /$ pulse is incident on an $11 \mathrm{~m}$ drift space in which about $10 \%$ of them decay. The decay products impinge on a two-dipole spectrometer optimized for very high rate operation. Straw chambers using fast gas constitute the first four planes where the rates are highest; conventional drift chambers are deployed in the rear. A novel feature is the beam plug which is designed to absorb the multi-GHz neutral beam part-way through the spectrometer. This eliminates acceptance-eating beam holes in the rear of the spectrometer and provides a quiet environment for the particle ID devices. These include an atmospheric hydrogen Cerenkov counter and a lead glass array for distinguishing electrons from heavier particles, and a muon range finder. The latter consists of planes of proportional wire chambers (and a few scintillator hodoscopes) at intervals of $5 \%$ in $\Delta p_{\mu} / p_{\mu}$. Muons are identified by the agreement of their momentum and range.

The second spectrometer magnet makes possible a redundant measurement of track momenta. The observed mass resolution for calibration $K_{L} \rightarrow \pi^{+} \pi^{-}$events is $\sigma_{m_{\pi \pi}}=1.13 \mathrm{MeV} / \mathrm{c}^{2}$. The net $p_{T}$ kick of the spectrometer is set to $220 \mathrm{MeV} / \mathrm{c}$, which tends to make the two-body decays of interest parallel after the last bend, facilitating a simple trigger. 


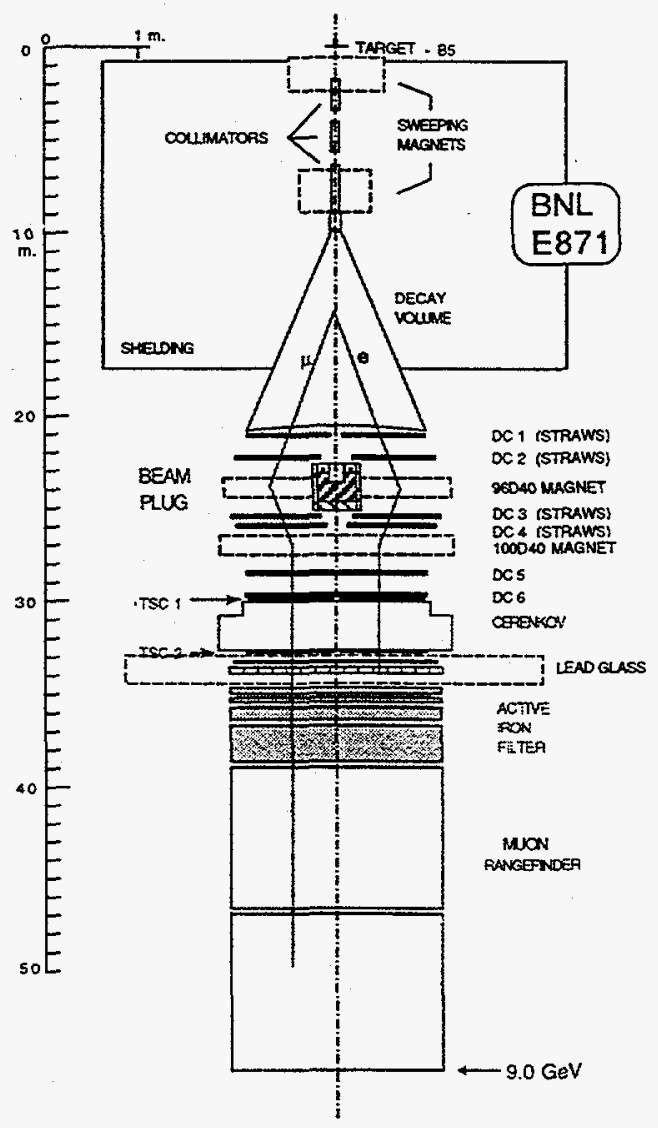

Figure 3: Apparatus of AGS E871.

The experiment had long data collection runs in 1995 and 1996. These runs were uncommonly eventful, and the experimenters had to contend with melted production targets, ruptured vacuum windows and other setbacks that would have confounded ordinary people. However, they prevailed and appear to have reached a sensitivity of $\sim 10^{-12}$ /event. Their analysis is in progress and results are expected within a few months. Fig. 4 shows the $m_{\mu \mu}$ distribution from a test analysis of a small sample of the 1995 data set. This demonstrates the power of the experiment to pull out a clear signal of a very rare $K_{L}$ decay $\left(B\left(K_{L} \rightarrow \mu^{+} \mu^{-}\right) \sim\right.$ $\left.7 \times 10^{-9}\right)$. The experimenters estimate they will reconstruct some $7000 K_{L} \rightarrow$ $\mu^{+} \mu^{-}$'s, to be compared to the present world sample of $\sim 1000$ events. This should yield a very accurate determination of the branching ratio, potentially allowing this decay to determine the CKM parameter $\rho$, modulo certain developments in theory and in experimental studies of other $K$ decays $^{7}$ (see Fig. 2).

Finally, the expected sensitivity of E871 is sufficient to observe the decay $K_{L} \rightarrow$ 


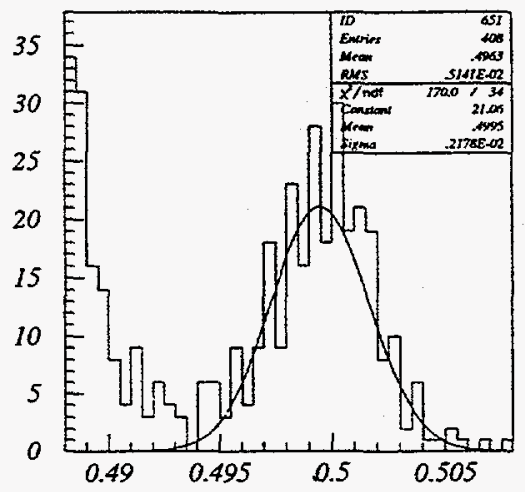

Figure 4: $K_{L} \rightarrow \mu^{+} \mu^{-}$signal from a small sample of AGS E871 data.

$e^{+} e^{-}$, which in the Standard Model is expected to occur at about $3 \times 10^{-12}$. This would be by far the rarest $K$ decay ever observed. Since the previous upper limit ${ }^{8}$ (from the predecessor experiment AGS-791) is $4.1 \times 10^{-11}$, there is approximately an order of magnitude in which surprises could lurk.

\section{$2.2 \quad \mathrm{E} 865$}

E865 is also primarily a search for LFV, in this case for $K^{+} \rightarrow \pi^{+} \mu^{+} e^{-}$. In this case a $6 \mathrm{GeV} / \mathrm{c}$ unseparated beam containing $70 \mathrm{MHz}$ of $K^{+}$and about 20 times more pions and protons is incident on a $5 \mathrm{~m}$ decay tank. Roughly $10 \%$ of the $K^{+}$decay in the tank and the resultant daughter tracks are momentum analyzed by the high rate proportional chamber spectrometer shown in Fig. 5 . The first magnet kicks the decay particles out of the hot beam region and separates them by sign (negative left). This allows the two sides of the detector to be separately optimized for particle identification. A great deal of attention has been paid to particle ID, since this is the most difficult challenge of the experiment. There are two layers of gas Čerenkov counters on each side of the detector. On the left, where it is essential that $\pi^{-}$'s are never mistaken for electrons (the worry is $K^{+} \rightarrow \pi^{+} \pi^{+} \pi^{-} ; \pi^{+} \rightarrow \mu^{+} \nu$ ), these counters are filled with hydrogen. On the right the $\check{\mathrm{C}}$ counters are filled with a lower threshold gas so that positrons are never mistaken for muons (the worry is $K^{+} \rightarrow \pi^{+} \pi^{0} ; \pi^{0} \rightarrow e^{+} e^{-} \gamma$ ) or for pions (the worry is $K^{+} \rightarrow \pi^{0} \mu^{+} \nu ; \pi^{0} \rightarrow e^{+} e^{-} \gamma$. Downstream of the last spectrometer element is a wall of lead-scintillator shower counters. It consists of some 600 $11.4 \mathrm{~cm} \times 11.4 \mathrm{~cm} \times 15$ r.l. elements read out via wavelength shifting fibers. Downstream of the calorimeter is a muon range stack of alternating proportional tube planes and steel absorber plates. 


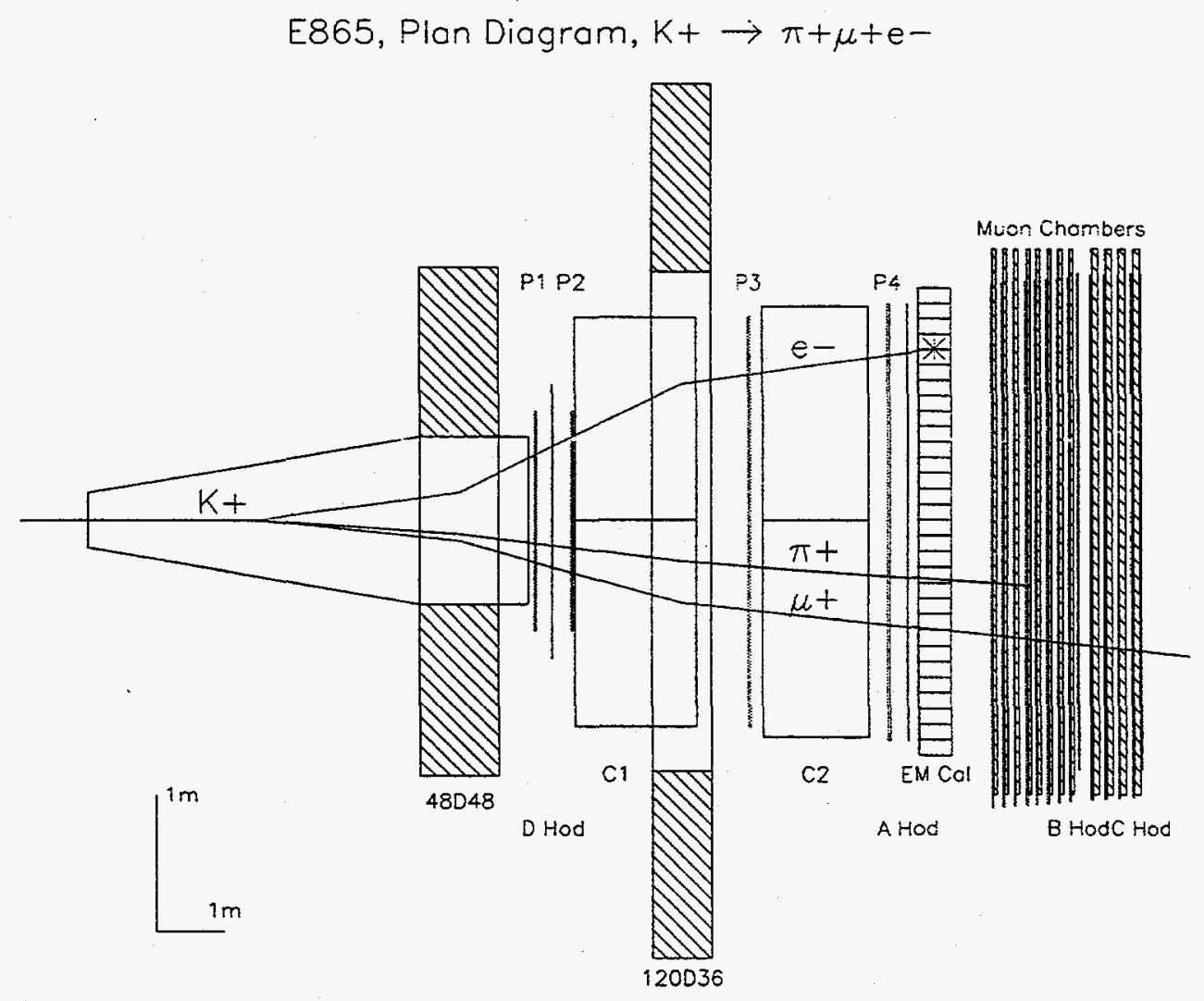

Figure 5: Apparatus of AGS E865.

E865 was commissioned in 1994 and has had significant data collecting runs in the subsequent two years. A sensitivity to $K^{+} \rightarrow \pi^{+} \mu^{+} e^{-}$of $\sim 10^{-11}$ /event can be expected from this data. This represents an improvement of an order of magnitude over the previous generation of this experiment. ${ }^{10}$ The goal of the experiment is to reach $\sim 10^{-12}$ /event sensitivity for $K^{+} \rightarrow \pi^{+} \mu^{+} e^{-}$and to study many other decay modes. Fig. 6 shows preliminary results from the 1995 run on one of these, $K^{+} \rightarrow \pi^{+} e^{+} e^{-}$. There are about twice as many events in this distribution as in the publication of the previous experiment, ${ }^{9}$ and many times this number are expected when E865 completes taking data. Using tagged $\pi^{0}$ 's from $K^{+} \rightarrow \pi^{+} \pi^{0}$ decay, data on $\pi^{0} \rightarrow e^{+} e^{-}$and $\pi^{0} \rightarrow e^{+} e^{-} e^{+} e^{-}$has also been collected.

The experiment has upgraded its DAQ system and is expected to accumulate sensitivity in 1997 at least $30 \%$ faster than in 1996. A scintillating pixel device for making beam measurements will be installed in 1997. This will permit the full reconstruction of decay modes with missing neutrinos. Along with improve- 


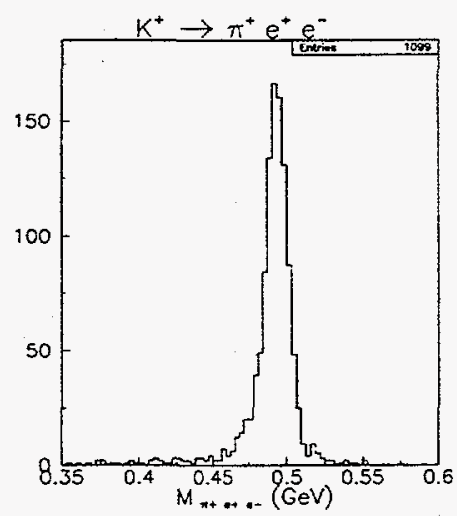

Figure 6: Effective mass of $\mathrm{K}^{+} \rightarrow \pi^{+} e^{+} e^{-}$candidates from E865.

ments to the particle ID and triggering, this should allow many additional decay modes to be studied. In 1997, E865 expects to collect data on $K^{+} \rightarrow \pi^{+} \mu^{+} \mu^{-}$, $K^{+} \rightarrow \mu^{+} \nu e^{+} e^{-}, K^{+} \rightarrow e^{+} \nu e^{+} e^{-}$, and $K^{+} \rightarrow \pi^{+} \pi^{0} e^{+} e^{-}$. These processes test the predictions of $\chi \mathrm{PT}$, and the last is a possible locus for CP-violation. This experiment may also take a special run to increase the world sample of $K^{+} \rightarrow \pi^{+} \pi^{-} e^{+} \nu$ events by an order of magnitude. This decay is of primary importance to $\chi \mathrm{PT}$, for which it provides much crucial input, and it is a primary source of information on low energy $\pi-\pi$ scattering.

\section{$2.3 \quad$ E787}

E787 is now primarily a search for the Standard Model process $K^{+} \rightarrow \pi^{+} \nu \bar{\nu}$. This highly GIM-suppressed decay can be very cleanly calculated in terms of SM parameters. As illustrated in Fig. 2, it is particularly sensitive to $\left|V_{t d}\right|$. The problematic hadronic matrix element that confounds efforts to extract $\left|V_{t d}\right|$ from $B-\bar{B}$ mixing is in this case determined through the well-known rate of $K e 3$ decay. ${ }^{11}$ Long distance contributions are negligible ${ }^{12}$ and QCD corrections are moderate and very well understood through the next to leading logarithmic order calculations of Buras and collaborators. ${ }^{13}$ The first generation ${ }^{14}$ of $E 787$ improved the upper limit on $B\left(K^{+} \rightarrow \pi^{+} \nu \bar{\nu}\right)$ from $1.4 \times 10^{-7}$ to $2.4 \times 10^{-9}$. CKM fits ${ }^{15}$ tend to predict a branching ratio of $<2 \times 10^{-10}$, so that there is still about an order of magnitude in which to search for new physics, for which predictions abound. ${ }^{3,4,16}$

$K^{+} \rightarrow \pi^{+} \nu \bar{\nu}$ has a far worse signature than most of the processes discussed above. Positive pions are very common decay products of $K^{+}$and there are no kinematic constraints other than $p_{\pi}<227 \mathrm{MeV} / \mathrm{c}$. To detect this process one basically has to prove one couldn't be seeing anything else. This entails excellent 
kinematic resolution, rejection of $\mu^{+}$by $\mathcal{O}\left(10^{9}\right)$, and the ability to veto extra particles by at least $10^{3}: 1 /$ particle. The only reason such an experiment is possible at all is that the primary background sources, $K^{+} \rightarrow \mu^{+} \nu$ and $K^{+} \rightarrow$ $\pi^{+} \pi^{0}$, are two body decays with very good signatures.

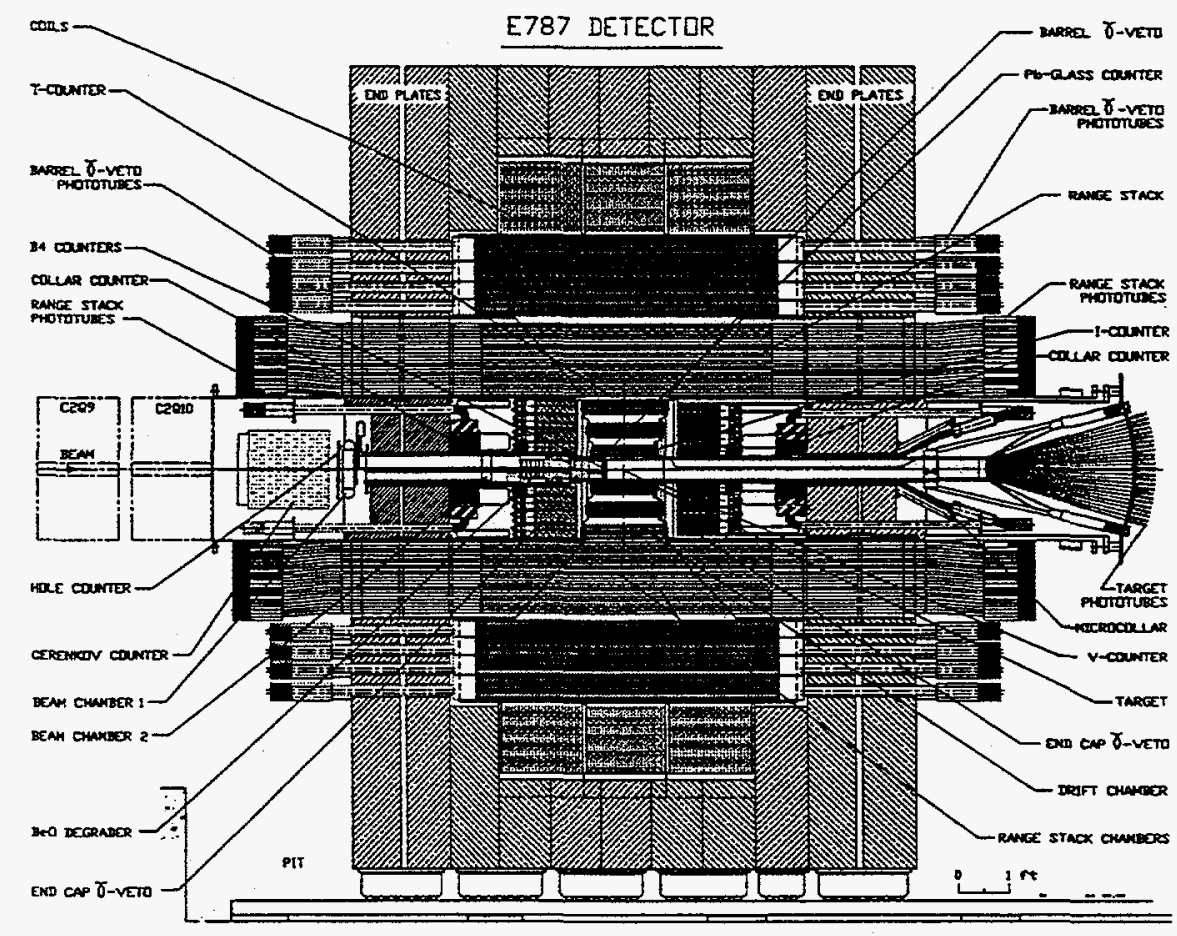

Figure 7: Apparatus of AGS E787.

Fig. 7 shows the detector ${ }^{17}$ which is mounted in a solenoidal magnet with a 2'-thick steel yoke. The necessity for hermetic vetoing and for excellent muon rejection have led all experiments hunting $K^{+} \rightarrow \pi^{+} \nu \bar{\nu}$ to employ a stopping geometry. An extremely pure $\sim 750 \mathrm{MeV} / \mathrm{c}$ beam containing about $10^{7} \mathrm{~K}^{+} / 1.6$ second spill is incident on a series of beam identification and tracking devices and loses most of its kinetic energy in a $\mathrm{BeO}$ degrader. The $K^{+}$stop in a highly segmented scintillating fiber target and the subsequent decay particles are momentum analyzed in a cylindrical drift chamber with thin-foil cathodes ${ }^{18}$ on which a 1-T magnetic field has been imposed. Candidate pions then range out in a cylindrical array of scintillators and straw chambers ("range stack"). This allows their kinetic energy and range to be measured. The range/energy/momentum comparison is a powerful means of particle ID in this energy range. The pions stop in range stack scintillators and their subsequent $\pi \rightarrow \mu \rightarrow e$ decay chain is monitored. This allows them to be distinguished from muons, whose decay chain contains only 
two pulses. Surrounding the range stack and plugging the ends of the device are photon detectors set in veto for $K^{+} \rightarrow \pi^{+}+$nothing events. In combination with the range stack these form a nearly hermetic photon veto.

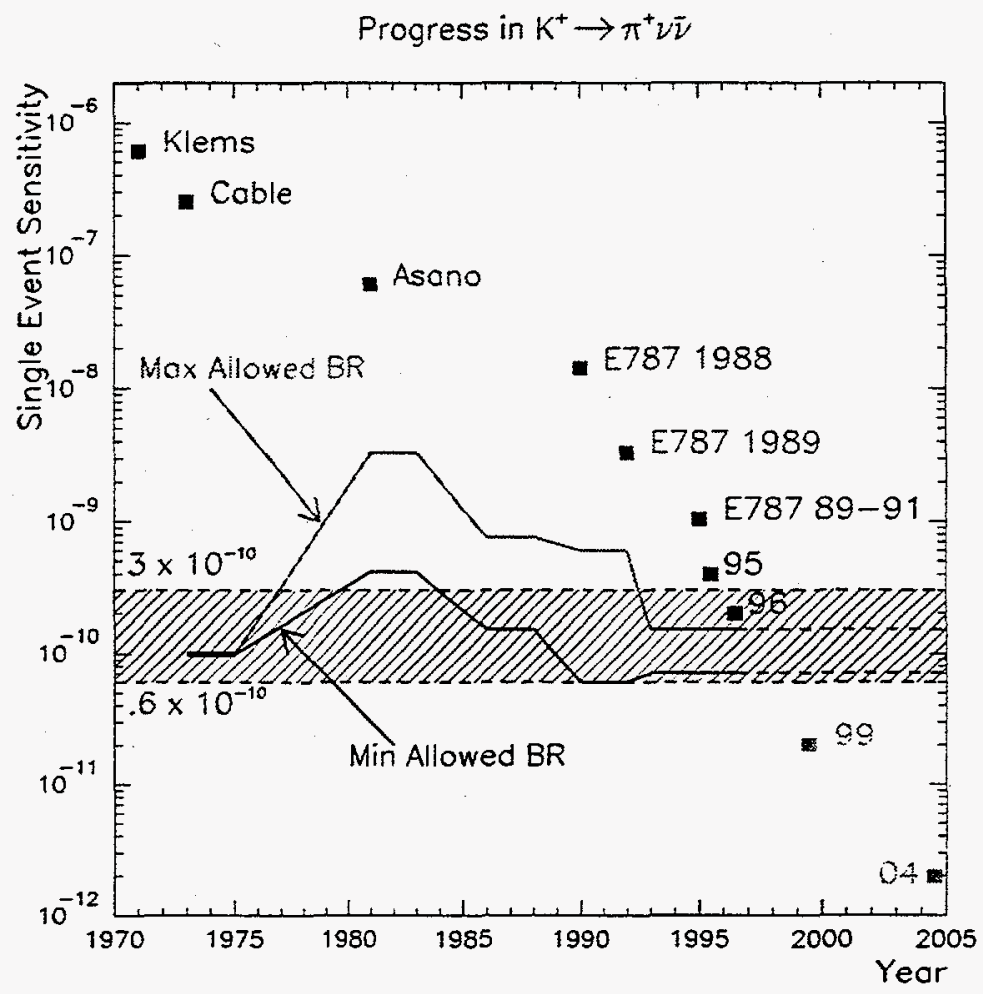

Figure 8: Progress of AGS E787. Hatched region is consistent with Standard Model. Solid lines show upper and lower limits of specific fits

The beam and much of the detector was upgraded in '91-94, and commisioned in 1994. Long data runs were taken in 1995 and 1996. Sensitivity corresponding to about 5 times that of all previous running of E787 was accumulated. Fig. 8 summarizes the situation. Data now in hand should yield a sensitivity of $\sim$ $2 \times 10^{-10}$ /event, compared with a central value for recent SM fits of $\sim 10^{-10}$. The limitations of the experiment are now reasonably well understood, and it is known that the background rejection power of the detector is sufficient to make a measurement at the predicted SM level. The most efficient way to improve the sensitivity of the experiment is to maintain the instantaneous rate of stopping 
$K^{+}$, but increase the duty factor. It is estimated that with long runs under optimum conditions, a sensitivity of $2 \times 10^{-11}$ /event could be achieved by 1999 . This corresponds to $\sim 5$ events at the central SM prediction. If, on the other hand, no events are observed at this level, the Standard Model must be wrong!

E787 has yielded quite a few interesting results on reactions other than $K^{+} \rightarrow$ $\pi^{+}+$nothing. I'll mention of couple of recent ones. The reaction $K^{+} \rightarrow \pi^{+} \gamma \gamma$ was initially important to E787 because of its potential as a background to $K^{+} \rightarrow$ $\pi^{+} \nu \bar{\nu}$. It is one of the very few possible SM sources of $\pi^{+}$stiffer than those from $K \rightarrow \pi^{+} \pi^{0}$ (i.e. in the main kinematic search region of E787). The pre-extant bound ${ }^{19}$ on $B\left(K^{+} \rightarrow \pi^{+} \gamma \gamma\right), 8.4 \times 10^{-6}$, was high enough to cause concern at the planning stage of $E 787$, before it was clear how well the photon veto would work. In fact, the the photon veto power turned out to be $\mathcal{O}\left(10^{3}: 1\right) / \gamma$ and, in an early measurement, ${ }^{20}$ E787 improved the upper limit on $B\left(K^{+} \rightarrow \pi^{+} \gamma \gamma\right.$ ) to $10^{-6}$ (and later ${ }^{21}$ to $\left.5 \times 10^{-7}\right)$ in the kinematic region of interest. This removed $K^{+} \rightarrow \pi^{+} \gamma \gamma$ from the list of significant backgrounds. However around that time, predictions ${ }^{22}$ of $\chi \mathrm{PT}$, indicated that this mode might be detectable by E787. These predictions indicated a very striking $m_{\gamma \gamma}$ distribution. Instead of the smooth phasespace-like distribution predicted by most previous models, there would be a sharp threshold at $m_{\gamma \gamma} \approx 2 m_{\pi}$. Crudely speaking, the idea is that the $K^{+}$decays virtually to three pions, and two of the pions annihilate into a $\gamma \gamma$ final state. The threshold in $m_{\gamma \gamma}$ corresponds to the region of soft $p_{\pi^{+}}$, which is difficult for E787 because of copious backgrounds from $K^{+} \rightarrow \pi^{+} \pi^{0}$ in which the $\pi^{+}$is down-shifted in momentum through interaction, and from $K^{+} \rightarrow \pi^{+} \pi^{0} \gamma$ and $K^{+} \rightarrow \pi^{+} \pi^{0} \pi^{0}$ where photons are lost. A careful analysis was successful in suppressing these backgrounds, and $\sim 30 \mathrm{~K}^{+} \rightarrow \pi^{+} \gamma \gamma$ events were observed. As seen in Fig.9, they exhibit the predicted threshold behavior.

A second recent result is on the radiative decay $K^{+} \rightarrow \mu^{+} \nu \gamma$. This reaction is dominated by inner bremsstrahlung (IB), and was first observed a number of years ago. However the primary physics interest in this decay is in the much rarer "structure dependent" radiation. A rich panoply of phenomena, including possible CP-violating interferences, have been predicted, but all previous attempts to see anything beside IB in this decay have produced only upper limits. ${ }^{23}$ In 1994 a short dedicated run was taken with the upgraded E787 detector. The trigger demanded both a stiff $\mu^{+}$and a single $\gamma$ with energy greater than that of most IB photons. Analysis revealed a clear structure-dependent component in the $K^{+} \rightarrow \mu^{+} \nu \gamma$ events collected. This can be seen in Fig. 10 where evidence for 


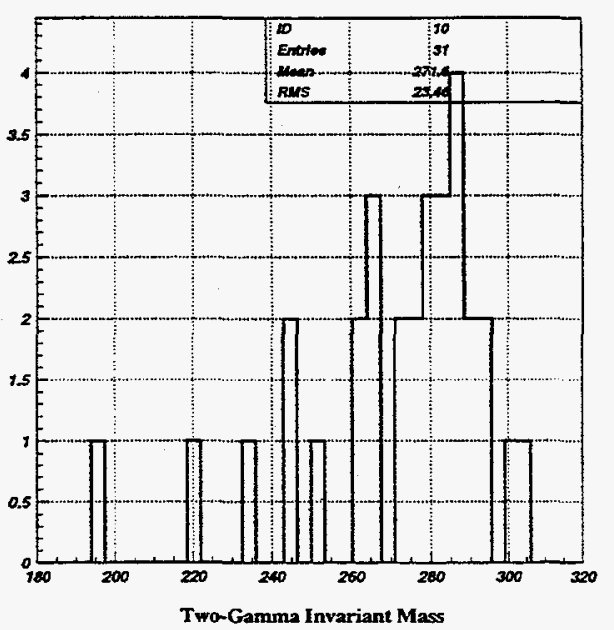

Figure 9: $m_{\gamma \gamma}$ distribution of $E 787 K^{+} \rightarrow \pi^{+} \gamma \gamma$ candidates.

$\sim 900$ non-IB events is exhibited.

A more sophisticated two dimensional analysis in which interference between various components of the decay was allowed yielded ${ }^{24} B\left(K^{+} \rightarrow \mu^{+} \nu \gamma\right)_{S D^{+}}=$ $(1.331 \pm 0.120 \pm 0.181) \times 10^{-5}$, where the first error is statistical and the second systematic. In terms of the vector and axial vector form factors, this corresponds to $\left|F_{V}+\dot{F}_{A}\right|=0.165 \pm 0.007 \pm 0.011$, which can be compared to the $\mathcal{O}\left(p^{4}\right) \chi \mathrm{PT}$ prediction $^{25} F_{V}+F_{A}=-.137 \pm 0.006$. Calculations to $\mathcal{O}\left(p^{6}\right)$ in $\chi \mathrm{PT}$ are in progress. ${ }^{26}$

\section{$3 \quad$ AGS-2000}

Although the AGS is slated to become an injector for RHIC in 1999, it will only be needed for this purpose for about two hours per day. The rest of the time it could be used for proton or heavy ion fixed target experiments. Since the base cost of maintaining the accelerator complex will be borne by RHIC, incremental running would be quite economical. To examine what experiments would be competitive in this situation, in May of 1996 the AGS-2000 Workshop was held at BNL. Experiments in many areas from muon decay to strangelet production were considered, but kaon decay was the most active subject. Table 4 lists the decays studied at the workshop. For lack of space, I will discuss only the first three of these. Details of the others may be found in the proceedings. ${ }^{27}$ 

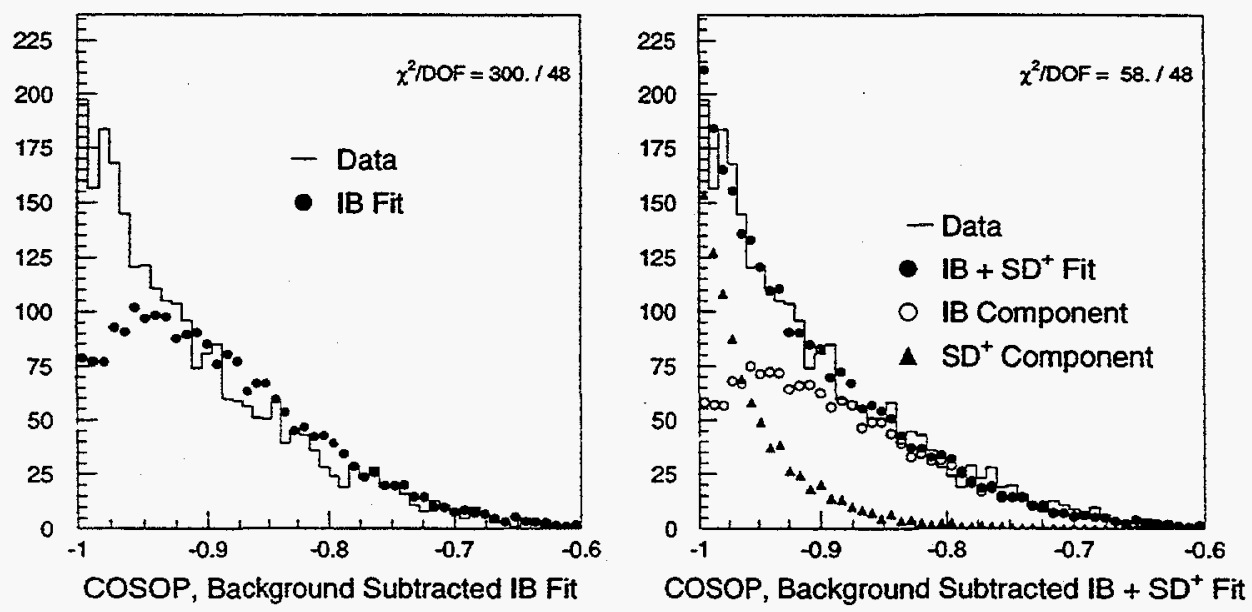

Figure 10: Distribution in $\cos \theta_{\mu \gamma}$ for $E 787 \mathrm{~K}^{+} \rightarrow \mu^{+} \nu \gamma$ candidates. Fit with IB alone is shown in (a), while fit with both $I B$ and structure-dependent radiation is shown in (b).

\section{$3.1 \quad K^{+} \rightarrow \pi^{+} \nu \bar{\nu}$}

A working group devoted itself to $K^{+} \rightarrow \pi^{+} \nu \bar{\nu}$ with the object of making a $15 \%$ measurement of $\left|V_{t d}\right|$, without building an entirely new detector. They concluded that this was possible if a higher acceptance, lower momentum $K^{+}$beam were built, the AGS macro duty factor optimized, the beam micro-bunched at $\sim 20$ $\mathrm{MHz}$ and certain straightforward improvements made to the E787 detector.

\section{$3.2 \quad K_{L} \rightarrow \pi^{0} \nu \bar{\nu}$}

As indicated in Fig. 2, the branching ratio for the decay $K_{L} \rightarrow \pi^{0} \nu \bar{\nu}$ is directly related $^{28}$ to CKM $\eta$. More explicitly ${ }^{29}$ for $m_{t}=175 \mathrm{GeV} / \mathrm{c}^{2}$,

$$
B\left(K_{L} \rightarrow \pi^{0} \nu \bar{\nu}\right) \approx 4.3 \times 10^{-10} \eta^{2} A^{4} .
$$

This corresponds to a predicted branching ratio of $\sim 2 \times 10^{-11}$. The theoretical situation is very clean: there are no significant long distance contributions, QCD corrections are small and well understood, the hadronic matrix element is known from $K e 3$, and the effect of indirect $\mathrm{CP}$ violation is $\mathcal{O}(1 \%)$. This reaction offers a window into the origin of $\mathrm{CP}$-violation that is comparable to studies proposed for the B-system and very complementary to them. ${ }^{29}$ It is also quite sensitive to new physics beyond the Standard Model. ${ }^{30} K_{L} \rightarrow \pi^{0} \nu \bar{\nu}$ is the most important measurement in the $K$ system and there are plans to pursue it in at least three laboratories. AGS-2000 stimulated a proposal ${ }^{31}$ to measure this process at BNL. 
Table 4: $K$ decays considered by AGS-2000.

\begin{tabular}{|l|l|}
\hline$K^{+} \rightarrow \pi^{+} \nu \bar{\nu}$ & precision measurement of $\left|V_{t d}\right|$ \\
\hline$K_{L} \rightarrow \pi^{0} \nu \bar{\nu}$ & clean measurement of CKM $\eta$ \\
\hline$K \mu 3$ & T-violating $\mu$ polarization tests non S.M. CP-violation \\
\hline$K_{L} \rightarrow \pi \pi \nu \bar{\nu}$ & clean measurement of CKM $\rho$ \\
\hline$K^{+} \rightarrow \pi^{+} \mu^{+} \mu^{-}$ & $\begin{array}{l}\text { P-violating } \mu^{+} \text {polarization measures } \rho, \\
\text { also sensitive to non-S.M. effects }\end{array}$ \\
\hline$K_{L} \rightarrow \mu^{+} \mu^{-}$ & Longitudinal $\mu$ polarization tests non S.M. CP-violation \\
\hline$K^{+} \rightarrow \pi^{+} \pi^{0} e^{+} e^{-}$ & $\begin{array}{l}\text { CP violation and form factor measurements, } \\
\text { sensitive to both S.M. \& non-S.M. CP-violation }\end{array}$ \\
\hline$K e 4$ & search for non-CKM CP violation \\
\hline$K e 3$ & determines $\left|V_{u s}\right|$ through an improved B.R. measurement \\
\hline
\end{tabular}

The present experimental upper limit ${ }^{32}$ on this decay is $5.8 \times 10^{-5}$, so that an increase in sensitivity of at least seven orders of magnitude is necessary in order to make a real measurement. The poor signature makes such a measurement at this level extremely challenging. Therefore it was proposed to measure everything possible about the reaction: the $K_{L}$ 's velocity as well as its direction, and also the directions of the final state photons (as well as their positions, energies, and arrival times). To measure the $K_{L}$ velocity, the AGS beam would be tightly bunched so that the time of flight origin of the $K_{L}$ could be known to $\sim 200$ ps. The beam can be microbunched on extraction by forcing it between empty RF buckets imposed by a dilution cavity. ${ }^{33}$ Tests of this technique in the AGS $^{34}$ have so far achieved bunch widths of $<300 \mathrm{ps}$ and are continuing.

To allow an accurate determination of the $K_{L}$ momentum from its velocity, the $K_{L}$ must be rather soft. To this end the beam is produced at a very large angle, i.e. $45^{\circ}$, so that the average $K_{L}$ momentum is $\sim 750 \mathrm{MeV} / \mathrm{c}$. Combined with good timing resolution on the final state photons, this allows a determination of $p_{K}$ to a few percent. The proposed beam is highly asymmetric with an aperture of $125 \mathrm{mr}$ horizontally and $4 \mathrm{mr}$ vertically, which facilitates collimation and affords an extra kinematic constraint. The proposed detector is shown in Fig. 11. The beam of $\sim 2.5 \times 10^{8} K_{L} /$ spill impinges on a $3.5 \mathrm{~m}$ decay tank. Decay photons are detected in a wall of directionalizing preconverters composed of $\sim 1.5 X_{0}$ of scintillators and low mass chambers. The energy measurement is completed by a lead-scintillating fiber calorimeter similar to that of the KLOE design ${ }^{35}$ but with a larger scintillator $/ \mathrm{Pb}$ ratio. The remainder of the acceptance, including 
the beam region, is covered by hermetic photon vetoes.

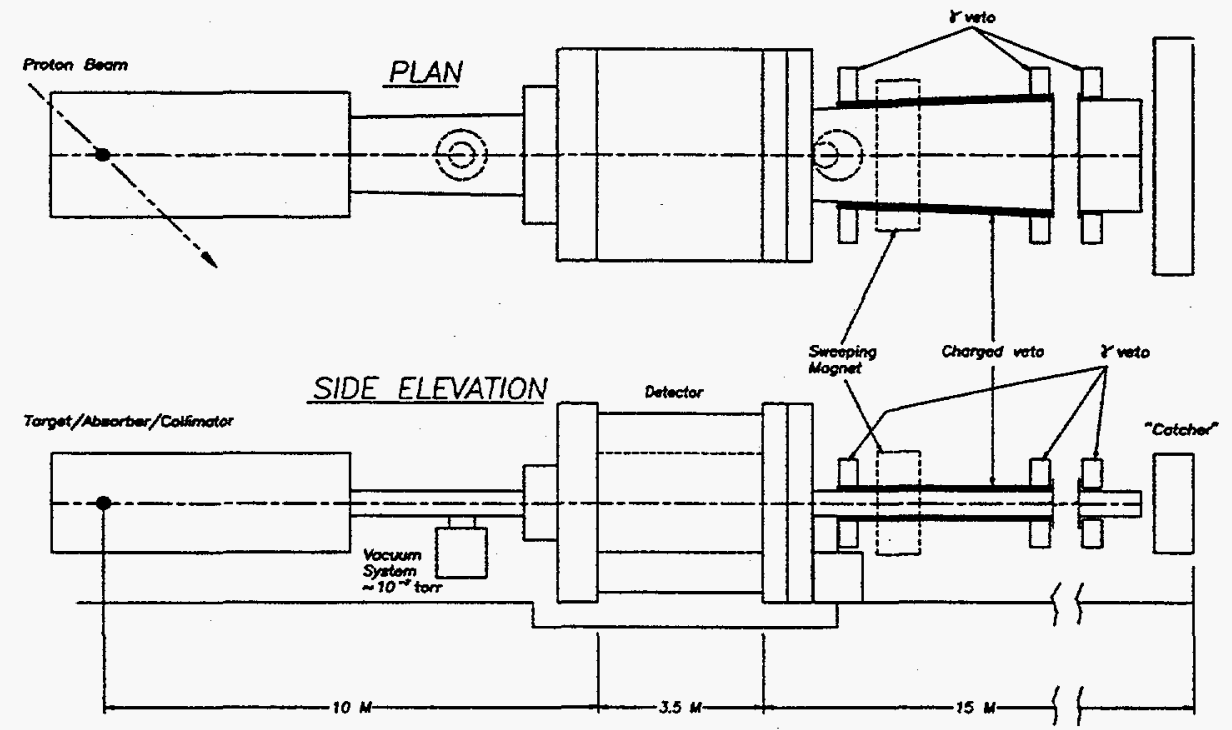

Figure 11: Proposed Apparatus of AGS E926.

The leading potential background to $K_{L} \rightarrow \pi^{0} \nu \bar{\nu}$ is $K_{L} \rightarrow \pi^{0} \pi^{0}$, which is almost $10^{8}$ times more copious than the signal. It is very difficult to veto $\pi^{0}$ to this level, hence the importance of the kinematic handle made possible by measuring the $K_{L}$ momentum. The $\pi^{0}$ 's from $K_{L} \rightarrow \pi^{0} \pi^{0}$ have a unique energy in the the $K_{L}$ center of mass system, and so can be eliminated kinematically. In addition to backgrounds due to $K$ decay, it is possible for a beam neutron to produce a $\pi^{0}$ ofl a residual gas molecule without any yielding any other visible decay product. This necessitates a vacuum of $10^{-7}$ Torr in the decay region, adding to the design challenge of this experiment.

In a three year run, the experiment would collect about $70 K_{L} \rightarrow \pi^{0} \nu \bar{\nu}$ events at the central Standard Model prediction, over a background of $\leq 10$ events. This would allow a determination of $\eta$ with a precision of $\leq 15 \%$. It would also be possible to get a limit on $K_{L} \rightarrow \pi^{0} \pi^{0} \nu \bar{\nu}$, a process which is cleanly sensitive to CKM $\rho .^{36,37}$ A number of other interesting decay modes could also be studied, including $K_{L} \rightarrow \gamma \gamma, K_{L} \rightarrow \pi^{0} \gamma \gamma$, and $K_{L} \rightarrow \pi^{0} \pi^{0} \gamma$.

\subsection{T-violation in $K^{+} \rightarrow \pi^{0} \mu^{+} \nu$ decay}

The present upper limit on T-violating muon polarization in $K \mu 3$ decay $\left(K^{+} \rightarrow\right.$ $\left.\pi^{0} \mu^{+} \nu\right),-0.009<P_{\mu}^{T}<0.007 @ 95 \%$ c.l., was established by a pair of BNL experiments $^{38}$ more than 15 years ago. Since the Standard Model predicts a negligible 


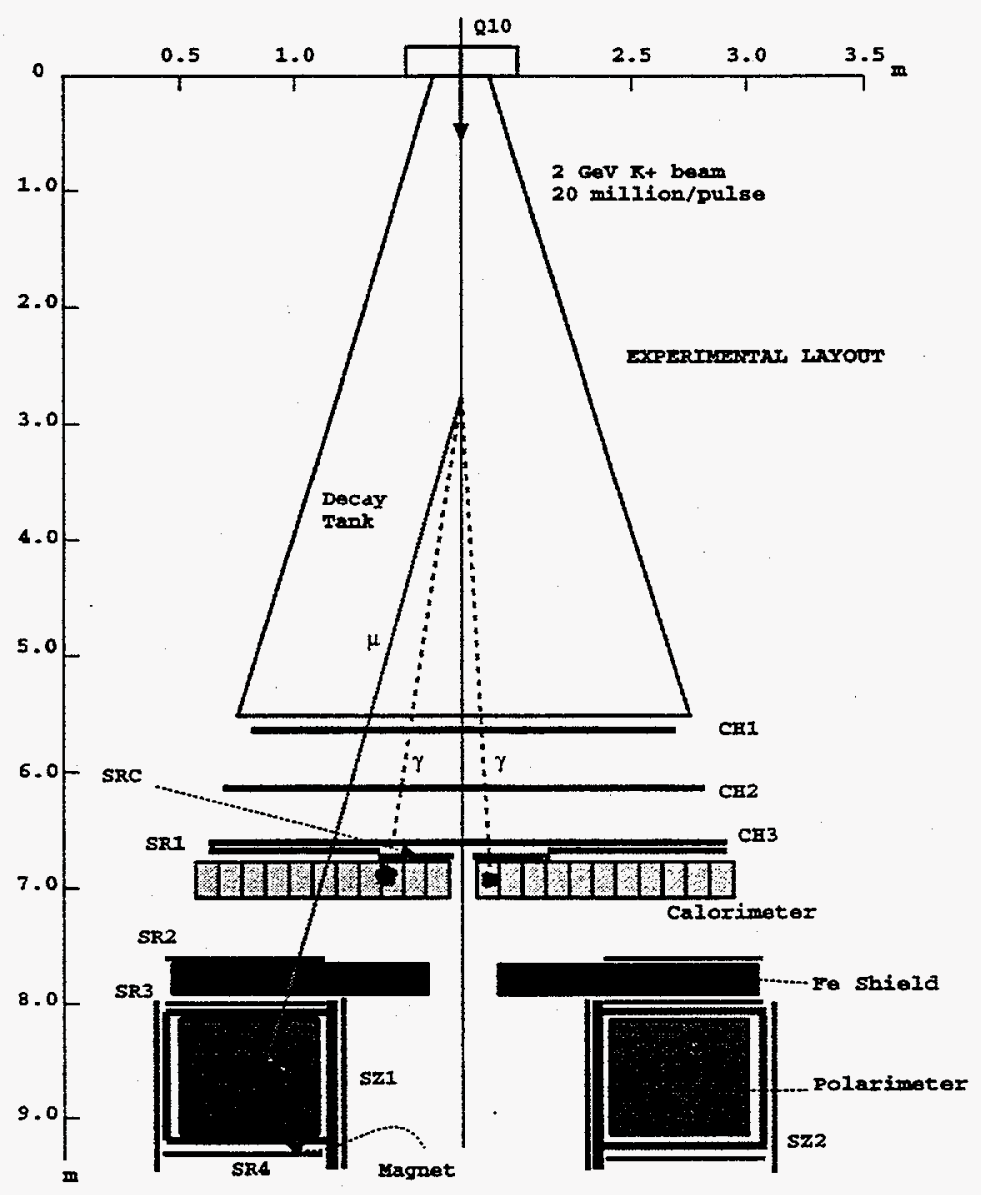

Figure 12: Proposed Apparatus of AGS E923.

effect, this reaction is a good place in which to search for BSM CP-violation. The observed baryon-antibaryon asymmetry of the universe appears to require sources of CP violation outside the SM, making this a very topical experiment. A number of such models, including those involving multiple Higgs $^{39}$ (in some cases arising from supersymmetry ${ }^{40}$ ) tend to predict detectable effects in $K^{+} \rightarrow \pi^{0} \mu^{+} \nu$. The present limits compare favorably with competing limits available from $B$-decays, so that any improvement in the $K$ limits would yield unique constraints on many models. At AGS-2000, a proposal ${ }^{41}$ to improve the sensitivity of this kind of experiment by about factor 40 was refined. The proposal attempts to capitalize on the experience of the earlier BNL experiment by retaining the same general philosophy, updated by advances in accelerator, beam, and detector technology. The previous $K^{+}$experiment was mounted in a $4 \mathrm{GeV} / \mathrm{c}$ unseparated positive beam. The current proposal utilizes the AGS $2 \mathrm{GeV} / \mathrm{c}$ separated beam, which can produce $2 \times 10^{7} K^{+} /$pulse with $K: \pi=1: 1.2$.

Fig. 12 shows the proposed apparatus. The beam is incident on a $\sim 5 \mathrm{~m}$ decay 
tank, in which roughly $5 \times 10^{6} \mathrm{~K}^{+} /$spill decay. The $\pi^{0}$ photons from $K \mu 3$ are detected in an electromagnetic calorimeter consisting of $\sim 500$ "Shashlyk" modules. Muons pass through a series of tracking chambers and trigger hodoscopes, and into a cylindrically symmetric polarimeter where they are stopped in radially arrayed graphite wedges. The decay of the stopped muons is detected by wire chambers that abut the absorber wedges. The chambers determine both the muon stop position and the positron direction. The trigger selects events in which the decay plane of the $K \mu 3$ coincides with the radial wedges. A $T$-violating muon polarization then manifests itself as an asymmetry between the number of muons whose decay positrons emerge in a clockwise sense and those whose decay positrons emerge in a counter-clockwise sense. A solenoidal field of $\sim 70$ gauss along the beam direction causes the muon spin to precess with a period of about $1 \mu \mathrm{sec}$. Reversing the polarity of the field every spill allows one to cancel many possible systematic errors that might otherwise allow the large in-plane component of polarization to cause a spurious signal.

The calculated analyzing power of the polarimeter is $\sim 35 \%$. Collecting 550 events/spill, in a 2000 hour run one can reach a statistical sensitivity of $1.3 \times 10^{-4}$ on $P_{\mu}^{T}$. This is equivalent to $\operatorname{Im} \xi \sim 7 \times 10^{-4}$, where $\operatorname{Im} \xi$ is the model independent $T$-violating amplitude. In terms of models such as those invoking three Higgs doublets, this is far more sensitive than existing limits from $B(b \rightarrow s \gamma)$ or $B(b \rightarrow$ $X \tau \nu)$.

\section{Conclusions}

The BNL Rare Kaon Decay Program has yielded a number of interesting discoveries and greatly improved the limits on several processes forbidden by the Standard Model. Experiments in progress now are expected to further improve these limits, and also to produce measurements of certain highly suppressed processes whose rates constrain important Standard Model parameters such as $\rho$ and $\left|V_{t d}\right|$. Future extensions of the program will be very economical to run after the AGS becomes an injector to RHIC. These could produce accurate measurements of the CKM $\mathrm{CP}$-violation parameter $\eta$, and probe BSM sources of CP-violation in unique ways. Since such experiments are typically sensitive to processes other than their primary quarry, they also offer an opportunity for the kind of completely unexpected discovery that has from time to time graced the history of kaon decay. 


\section{Acknowledgments}

I would like to thank D. Bryman, A. Buras, M. Diwan, S. Kettell, H. Ma, W. Molzon, J. Ritchie, and M. Zeller for discussions, corrections, access to data and other assistance with this paper. This work was supported by the U.S. Department of Energy under Contract No. DE-AC02-76CH00016.

\section{References}

[1] J. Ritchie and S. Wojcicki, Rev. of Mod. Phys. 651149 (1993).

[2] W. Buchmüller and D. Wyler, Nucl. Phys. B268 621 (1986); R. Cahn and H. Harari, Nucl. Phys. B176 135 (1980); J. Pati and H. Stemnitzer, Phys. Lett. 172B 441 (1986): O. Shanker, Nucl. Phys. B206 253 (1982); A. Acker and S. Pakvasa, Mod. Phys. Lett. A7 1219 (1992); W.J. Marciano and A. Sanda, Phys. Rev. Lett. 381512 (1977); W.J. Marciano, Phys. Rev. D45 R721 (1992); A. Barroso, G. Branco and M. Bento, Phys. Lett. 134B 123 (1984); P. Langacker, S. Uma Sankar and K. Schilcher, Phys. Rev. D38 2841 (1988).

[3] I. Bigi and F. Gabbiani, Nucl. Phys. B367 3 (1991).

[4] J. Hagelin and L. Littenberg, Prog. Part. Nucl. Phys. 231 (1989).

[5] Y. Asano et. al., Phys. Lett. 107B 159 (1981).

[6] A. Pich,Rept. Prog. Phys. 58563 (1995).

[7] L.Littenberg and G. Valencia, Ann. Rev. Nucl. Part. Sci. 43729 (1993).

[8] K. Arisaka et. al., Phys. Rev. Lett. 713910 (1993).

[9] C. Alliegro et. al, Phys. Rev. Lett. 68278 (1992).

[10] A. M Lee et al., Phys. Rev. Lett. 64165 (1990).

[11] W.J. Marciano and Z. Parsa, Phys. Rev. D53 1R (1996).

[12] D. Rein and L.M. Sehgal, Phys. Rev. D39 3325 (1989); M. Lu and M. B. Wise, Phys. Lett. B324 461 (1994); C.Q. Geng, I.J. Hsu, and Y.C. Lin, Phys.Lett. B55 569 (1995).

[13] G. Buchalla and A.J. Buras, Nucl. Phys. B412 106 (1994).

[14] S. Adler et al., Phys. Rev. Lett. 761421 (1996).

[15] S.Herrlich and U. Nierste, Phys. Rev. D52 6505 (1995). 
[16] F. Wilczek, Phys. Rev. Lett. 491549 (1982).

[17] M. Atiya et al., Nucl. Inst. \& Meth. A321 129 (1992).

[18] E.W. Blackmore et al., submitted to Nucl. Inst. E Meth. A (1996).

[19] Y. Asano et al., Phys. Lett. 113B 195 (1982).

[20] M.S. Atiya et al., Phys. Rev. Lett. 651188 (1990).

[21] S. Adler, "The Search for the $K^{+} \rightarrow \pi^{+} \gamma \gamma$ Decay Above the $K_{\pi 2}$ Peak Using the 1991 Data Set", State University of New York at Stony Brook thesis (1995).

[22] G. Ecker, A. Pich and E. de Rafael, Nucl. Phys. B303 665 (1988);G. Ecker, A. Pich and E. de Rafael, Phys. Lett. 237B, 481 (1990).

[23] Y. Akiba et al., Phys. Rev. D32 2911 (1985); V.S. Demidov et al.,Sov. Jour. Nucl. Phys.52 1006 (1990).

[24] M. Convery, First Measurement of Structure Dependent $K \rightarrow \mu^{+} \nu \gamma$, Princeton University $\mathrm{PhD}$. thesis (1996).

[25] J. Bijnens, G. Ecker, and J. Gasser, Nucl. Phys. B396 81 (1993).

[26] Ll. Amettler, et al., Phys. Lett. B303 140 (1993).

[27] AGS-2000 Experiments for the 21st Century L.S. Littenberg and J. Sandweiss. Proc., AGS-2000 Workshop, Brookhaven National Laboratory, May 13-17, 1996, Formal Report.

[28] L. Littenberg, Phys. Rev. D39 3322 (1989).

[29] G. Buchalla and A.J. Buras, Phys. Rev. D54 6782 (1996).

[30] Y. Grossman and Y. Nir, SLAC-PUB-7380, Jan. 1997, hep-ph/9701313.

[31] I-H. Chiang, et al., "Measurement of $K_{L} \rightarrow \pi^{0} \nu \bar{\nu}$ ", AGS Proposal 926 (1996).

[32] M.B. Weaver, et al., Phys. Rev. Lett. 72, 3758 (1994).

[33] R. Cappi and C. Steinbach, CERN-PS-OP-81-10, Particle Accelerator Conference 1981, 2806.

[34] J. W. Glenn, AGS/AD Technote 426 (1996).

[35] A. Antonelli, et al., N.I.M. A354 352 (1995).

[36] C.Q. Geng, I.J. Hsu, and Y.C. Lin, Phys.Rev. D50 5744 (1994).

[37] L. Littenberg and G. Valencia, Phys. Lett. 385B 379 (1996). 
[38] M. Schmidt, et al., Phys. Rev. Lett. 43, 556 (1979); W. Morse, et al., Phys. Rev. D21, 1750 (1980); M. Campbell, et al., Phys. Rev. Lett. 47, 1032 (1981); S. Blatt, et al., Phys. Rev. D27, 1056 (1983).

[39] R. Garisto and G. Kane, Phys. Rev. D44, 2038 (1991); G. Belanger and C. Q. Geng, Phys. Rev. D44, 2789 (1991).

[40] G-H. Wu and J. Ng,TRI-PP-96-57, Oct 1996, to be published in Phys. Rev..

[41] M.V. Diwan, et al., "Search for T-Violating Muon Polarization in $K^{+} \rightarrow$ $\mu^{+} \pi^{0} \nu_{\mu}$ Decay", AGS Proposal 923 (1996). 